\title{
Developing an Userfriendly Online Shopping Web-Site
}

\author{
G. Saibaba, S. Prasanth Vaidya \\ Computer Science and Engineering, Gayatri Vidya Parishad College of Engineering, \\ Madhurawada, Visakhapatnam, Andhra Pradesh 530048, India
}

\begin{tabular}{l}
\hline \hline Article Info \\
\hline Article history: \\
Received May 1, 2018 \\
Revised Jun 13, 2018 \\
Accepted Aug 21, 2018
\end{tabular}

Keywords:

Online shopping

E-commerce Website

Web Application

\begin{abstract}
In this era of internet, e-commerce is growing by leaps and bounds keeping the growth of brick-and-mortar businesses in the dust. In many cases, brickand-mortar businesses are resorting to having a counterpart which is internet or e-commerce driven. People in the developed world and a growing number of people in the developing world now use ecommerce websites on a daily basis to make their everyday purchases. Still the proliferation of e-commerce in the underdeveloped world is not that great and there is a lot to desire for It consists of the planning process, which starts with determining the use case, domain modeling and architectural pattern of the web application. The entire development process is primarily divided into two parts: the frontend development and the back end development. The database design is also discussed with an emphasis on its relational connectivity.
\end{abstract}

Copyright $@ 2018$ Institute of Advanced Engineering and Science. All rights reserved.

\section{Corresponding Author:}

S. Prasanth Vaidya,

Computer Science and Engineering,

Gayatri Vidya Parishad College of Engineering,

Madhurawada, Visakhapatnam,

Andhra Pradesh 530048, India. +91-9652733636

Email: vaidya269@gmail.com

\section{INTRODUCTION}

Electronic commerce or e-commerce refers to a comprehensive range of online business activities for prod- ucts and services. It is generally accompanying with online buying and selling over the internet or guidance any transaction involving the transpose of ownership or rights to employment goods or services through a computer mid- dle network $[1,2]$. E-commerce is dividing into three categories: business to business or B2B (Cisco), business to consumer or B2C (Amazon), and consumer to consumer or C2C (eBay).

\subsection{Background}

E-commerce website development is done using front-end and back-end development. Front-end develop- ment can be done using HTML, CSS, javascript, jquery and Ajax. HTML is the standard for hyper text markup language for creating Web pages. It is mainly used to describe the structure of Web pages with elements. Each elements contains start and end tags. CSS is Cascading Style Sheets it mainly used to describe how HTML elements are to be displayed on screen. It can control the layout of multiple web pages all at once [3, 4]. Chandran et al. [5] proposed website quality evaluation based on search engine queries using web rank position algorithm. Zhang et al. [6] proposed E-commerce website recommender system based on dissimilarity and association rule. Qui et al. [7] designed website resource monitoring platform supporting tibetan and uyghur language based on semantics.

\subsection{The Problem}

Providing sales and advertisement for small scale merchandiser is a very difficult task. Due to dayto-day competetion the seller's are facing many problems to sell the products to the customers. In the similar 
way, the customer's required easy shopping facilities and multiple products which are present at the same place.

\subsection{The proposed solution}

To overcome the difficulties of the sellers and the consumers, e-commerce userfriendly website is designed with a very low budget where the conusumers can select the product and purchase it at their home. Similarly the sellers can provide all the products in a single window.

Model-view-controller is commonly used for developing software that divides an application into three inter- connected parts. The model is the central component of the pattern. It expresses the application's behavior in terms of the problem domain, independent of the user interface. A view can be any output representation of information, such as a chart or a diagram. Multiple views of the same information are possible, such as a bar chart for management and a tabular view for accountants. The third part or section, the controller, accepts input and converts it to commands for the model or view [8].

In addition to dividing the application into three kinds of components, the model-view-controller design defines the interactions between them. The model is responsible for managing the data of the application. It receives user input from the controller. The view means presentation of the model in a particular format. The controller responds to the user input and performs interactions on the data model objects. The controller receives the input, optionally validates it and then passes the input to the model [9].

In the proposed model, setting up a website for business puropose is addressed from both the owner and the consumer point of view. Since the evaluation is based on the owners process i.e., creating a website, understanding consumer requirements and so on. The rest of the paper is is organized as follows. In Section 2, features of the model are discussed. In Section 3, the planning process is discussed. In Section 4, the tools required for developing the model are presented. The working of the given model is presented in Section 5. In Section 6, testing and bug fixing are discussed. Finally, the conclusion is provided in Section 7.

\section{FEATURES}

An end user can achieve free online registration and can search a specific product of his/her interest. The payment can be done online or through "Cash on delivery" manner. The administrator has the right to add product, update its cost or cancel any product. Customers can update their personal inforamtion any time. After logging into the system, the customers can order the required product. In this ecommerce website, products are organized based on categories. Whenever a customer purchases a product, it automatically subtracts the count from the inventory system \& if the quantity of the product is less than five, notification will be sent to the admin and supplier [10].

\section{THE PLANNING PROCESS}

The goal is to disentangle a web application that would be attractive enough, have a professional look and user favorable. So that, people of all period groups would be its end users which begin with subdividing the whole task and setting milestones. The entire planning process is discussed here.

\subsection{Defining Use Case Models}

Writing use cases or stories of using a system is an excellent technique to explain and describe requirements. An end user with internet browsing facility can register into the site. By signing in, they can find all the products provided; users can find the products using the search option. Items to be purchased are added into the shopping cart. By providing payment type, the order will be completed bygenerating e-bill. The following are the primary requirements

1) A registration page

2) Search option

3) Shopping cart

4) Admin page

5) Payment page and so on.

\subsection{Domain Modeling}

The proposed web application model is developed using Object Oriented Programming (OOP) which em- phasizes on finding and describing the objects. 


\subsection{Architectural Pattern}

The Proposed application has been improved using standard "Model-View-Controller" pattern. Model view controller (MVC) is an application architectural pattern for implementing user interfaces. MVC is an open source web development framework that provides support to build the application using PHP with MVC pattern. It can only pass information from the view and updated information to the view. Finally, the model can only access to mysql database where it updates login information, registration pages, prices and products entered by the admin or the user. The model, view and controller of the application is shown in Figure 1 with user view and admin view consisting of login, header, footer and so on, model consisting of login, product, category and so on and controller for both admin and user to manipulate and update user and admin view with the help of the model.

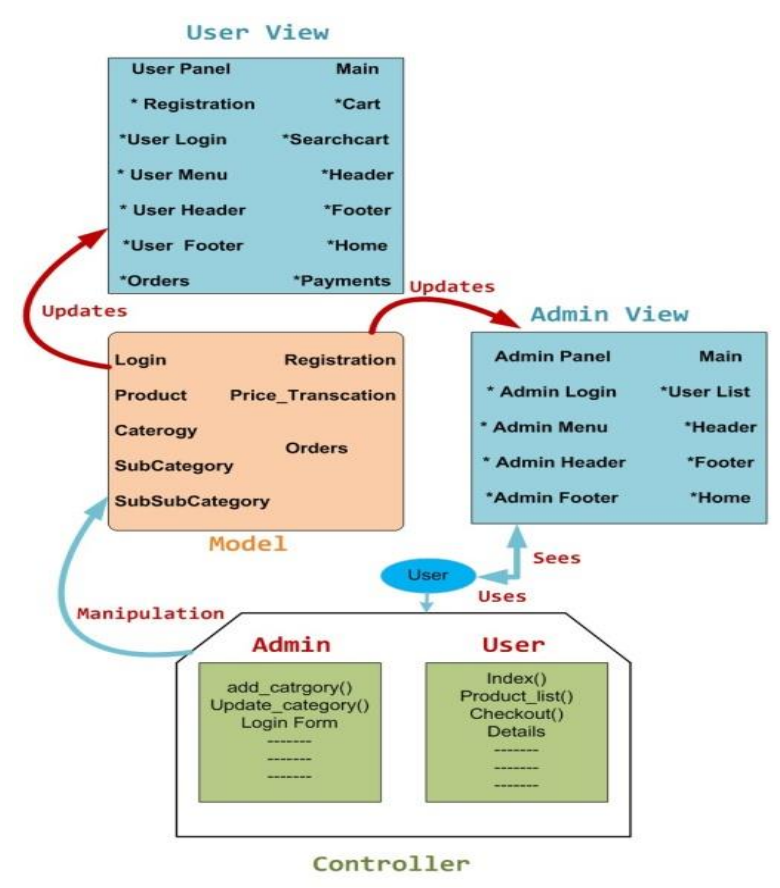

Figure 1. Diagram of Model, View and Controller of the application

\section{DEVELOPMENT TOOLS}

The entire development process has been sub-divided into front-end and back-end development. The front- end development consists of visually visible on the application, such as home page, admin panel, contact page, shop- ping cart page and so on. The back-end development contains my-sql-database and its interaction with front-end pages.

\subsection{Front-End Development}

Front-end development mainly using HTML, CSS, Java Script, JQuery and Ajax.

\subsubsection{Hypertext Mark-up Language (HTML)}

HTML is the standard for hyper text markup language for creating Web pages. It is mainly used to describe the structure of Web pages. The document contains two section first section is header section and second is body section [11]. The header sections contain cascading style sheets and javascript files. The body section contains visible part of the web applications with elements (start and end tags) [12, 13].

\subsubsection{Cascading Style Sheets (CSS)}

CSS is Cascading Style Sheets it mainly used to describe how HTML elements are to be displayed on screen [14]. CSS is a style sheet language used for describing the look and formatting a document written in a mark- up language. These CSS files are linked with the class files with .php extensions to put the panels in order, the text with correct font, size and colour. CSS can control the layout of multiple web pages all at once [15]. 


\subsubsection{Java Script, JQuery \& Ajax}

Java Script is a client side scripting language, most commonly used as part of web browsers. It's implementations allow client side scripts to interact with the user, control the browser, communicate asynchronously and alter the document content that is displayed. JavaScript is scripting languages it mainly used to makes HTML pages more dynamic and interactive. JQuery is a JavaScript Library. The purpose of JQuery is to make it much easier to use JavaScript on the website [16, 17]. JQuery takes a lot of common tasks that require many lines of JavaScript code to accomplish, and wraps them into methods that can call with a single line of code. JQuery also simplifies a lot of the complicated things from JavaScript, like AJAX calls and DOM manipulation. AJAX is Asynchronous JavaScript and XML, AJAX allows to send and receive data asynchronously without reloading the web page [18].

\subsection{Backend Development}

Database Management System (DBMS) provides support for back-end development. DBMS is essentially software to create the database to add, drop, alter and update tables [19]. Back-end development uses PHP with mysql. PHP is hyper text preprocess which is mainly used to create dynamic webpage. PHP can create, open, read, write, delete, and close files on the server which contains text, HTML, CSS and PHP Code $[20,21]$.

\subsection{Database Design}

One of the most important and challenging task is database design. The information passed by the users while registering in the application is stored in the mysql database. The items with their identification, description and image are stored in the database. Tables in the proposed application database are: registration, userlogin, admin login, category, subcategory, subsubcategory, items, wishlist, orders, logs and so on. The formation of three tables are shown in the Figure 2. The table entitled "product" has the attributes namely: producti d, productr ate, categoryi $\mathrm{d}$, description and status where producti $\mathrm{d}$ is the primary key for this table i.e., each product will have a unique identity. The attribute categoryi d plays as a foreign key for this table i.e. this foreign key creates a link with table category having two attributes: categoryi d and categoryn ame. When a customer registers in the website, information will be stored in the customer table with the attributes name, customeri d, producti d, quantity and email. Again customeri dis the unique key for this table and producti $d$ is the foreign key to link with the product table.

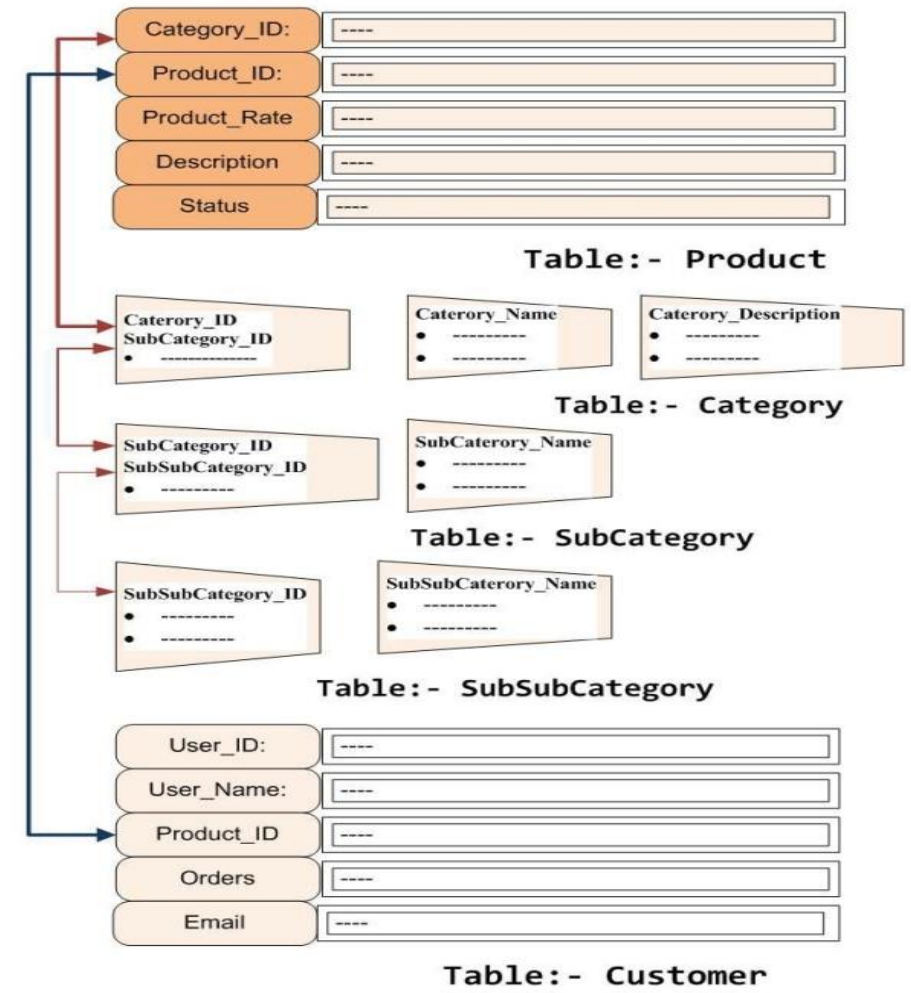

Figure 2. Table of product, category, subcategory, subsubcategory and customer database 


\section{HOW THE WEB APPLICATION WORKS}

The proposed web application comprises of dynamic web pages which has been created for both client and server side scripts. A dynamic web page is a web page that is generated by a server-side program or script. For the testing purpose website is hosted on local hosts i.e., personal computers. Browser like Google Chrome, Mozilla Firefox or Safari support this program. The browser makes a Hypertext Transfer Protocol (HTTP) request to the web server for a specific dynamic web page, the web server then looks up the extension of the requested file to find out which application server should process the request. When the application server receives a request, it runs the specified script. Often, this script uses the data that it gets from the web browser to get the appropriate data from a database server. This script can also store the data that it receives in the database. When the application server finishes processing the data, it generates the HTML for a web page and returns to the web server. Then, the web server returns the HTML to the web browser as part of an HTTP response

\section{TESTING AND BUG FIX}

Testing technique included the process of executing the application with the intent of finding bugs (errors or other defects). It involved execution of the most important application components to evaluate the properties of interest. Among the several testing methods White-box testing was preferred. White-box testing also known as clear box testing, tests internal structures or workings of a program, as opposed to the functionality exposed to the end user. In white box testing an internal perspective of the system as well as programming skills are used to design test cases. The tester chooses inputs to exercise paths through the code and determine the appropriate outputs. This is analogous to testing nodes in a circuit. Based on the above test, it can stated that the proposed web application is perfectly working. There were few bugs later fixed and now it is a full functioning website. To ensured the security issues, cryptographic hash functions like SHA-2 is used during the implementation of the functions where the application asks the user for a password.

\section{CONCLUSION}

E-Commerce has traversed the life styles entirely by providing easy purchasing options without going out. The seller can readily start online business with the help of e-commerce application development and web development solutions. It is one of the cheapest means of doing business by providing promotions to products and services with less cost without time barriers. An interactive, user friendl and secured website is generated for online shopping.

\section{REFERENCES}

[1] M. Niranjanamurthy, N. Kavyashree, S. Jagannath, and D. Chahar, "Analysis of e-commerce and m-commerce: advantages, limitations and security issues," International Journal of Advanced Research in Computer and Communication Engineering, vol. 2, no. 6, 2013.

[2] A. Sivaji, A. G. Downe, M. F. Mazlan, S.-T. Soo, and A. Abdullah, "Importance of incorporating fundamental usability with social \& trust elements for e-commerce website," in Business, Engineering and Industrial Applications (ICBEIA), 2011 International Conference on. IEEE, 2011, pp. 221-226.

[3] S. Gupta, "Online shopping cart application," 2013.

[4] M. C. Buzzi, M. Buzzi, B. Leporini, and F. Akhter, "User trust in ecommerce services: perception via screen reader," in New Trends in Information and Service Science, 2009. NISS'09. International Conference on. IEEE, 2009, pp. 1166-1171.

[5] M. Chandran et al., "Website quality evaluation based on search engine queries using web rank position algorithm (wrpa)," Indonesian Journal of Electrical Engineering and Computer Science, vol. 4, no. 1, pp. 224-230, 2016.

[6] L. Zhang, S. Yang, and M. Zhang, "E-commerce website recommender system based on dissimilarity and association rule," Indonesian Journal of Electrical Engineering and Computer Science, vol. 12, no. 1, pp. 353-360, 2014.

[7] L. Qiu, "Website resource monitoring platform supporting tibetan and uyghur language based on semantics," Indonesian Journal of Electrical Engineering and Computer Science, vol. 11, no. 8, pp. 4766-4773, 2013.

[8] V. Pujani, "Use of ecommerce websites in developing countries," World Academy of Science, Engineering and Technology, vol. 78, pp. 790-795, 2011.

[9] S. Sharma, "Internet marketing: The backbone of ecommerce," International Journal of Emerging Research in Management \&Technology, vol. 4, no. 12, pp. 200-202, 2015.

[10] A. B. Mathew, R. Ravi, S. Mohan, and T. Ramakrishnan, "E-commerce online purchasing based on comparison."

[11] S. Gupta, G. Kaiser, D. Neistadt, and P. Grimm, "Dom-based content extraction of html documents," in Proceedings of the 12th international conference on World Wide Web. ACM, 2003, pp. 207-214.

[12] C. Musciano, B. Kennedy et al., HTML, the definitive Guide. O’Reilly \& Associates, 1996. 
[13] D. Raggett, A. Le Hors, I. Jacobs et al., "Html 4.01 specification," W3C recommendation, vol. 24, 1999.

[14] O. Rubio-Cabezas, V. Puri, I. Murano, V. Saudek, R. K. Semple, S. Dash, C. S. Hyden, W. Bottomley, C. Vigouroux, J. Magre' et al., "Partial lipodystrophy and insulin resistant diabetes in a patient with a homozygous nonsense mutation in cidec," EMBO molecular medicine, vol. 1, no. 5, pp. 280-287, 2009.

[15] M. Ahmadian, M. J. Abbott, T. Tang, C. S. Hudak, Y. Kim, M. Bruss, M. K. Hellerstein, H.-Y. Lee, V. T. Samuel, G. I. Shulman et al., "Desnutrin/atgl is regulated by ampk and is required for a brown adipose phenotype," Cell metabolism, vol. 13, no. 6, pp. 739-748, 2011.

[16] D. Flanagan, JavaScript: the definitive guide. " O'Reilly Media, Inc.”, 2006.

[17] D. Crockford, "The application/json media type for javascript object notation (json)," 2006.

[18] T. Bray, "The javascript object notation (json) data interchange format," 2017.

[19] D. Maier, J. Stein, A. Otis, and A. Purdy, Development of an object-oriented DBMS. ACM, 1986, vol. 21, no. 11.

[20] M. Stonebraker and D. Moore, "Object-relational dbms-the next wave," Informix Software (now part of the IBM Corp. family), Menlo Park, CA, p. 14, 1995.

[21] M. Stonebraker, D. J. Abadi, A. Batkin, X. Chen, M. Cherniack, M. Ferreira, E. Lau, A. Lin, S. Madden, E. O’Neil et al., "C-store: a column-oriented dbms," in Proceedings of the 31 st international conference on Very large data bases. VLDB Endowment, 2005, pp. 553-564. 